\title{
Dynamic VO Establishment in Distributed Heterogeneous Business Environments
}

\author{
Bartosz Kryza $^{1}$, Lukasz Dutka ${ }^{1}$, Renata Slota ${ }^{2}$, and Jacek Kitowski ${ }^{1,2}$ \\ ${ }^{1}$ Academic Computer Centre CYFRONET-AGH, Cracow, Poland \\ ${ }^{2}$ Institute of Computer Science, AGH-UST, Cracow, Poland \\ \{bkryza, dutka, rena, kito\}@agh.edu.pl
}

\begin{abstract}
As modern SOA and Grid infrastructures are being moved from academic and research environments to more challenging business and commercial applications, such issue as control of resource sharing become of crucial importance. In order to manage and share resources within distributed environments the idea of Virtual Organizations (VO) emerged, which enables sharing only subsets of resources among partners of such a VO within potentially larger settings. This paper describes the Framework for Intelligent Virtual Organizations (FiVO), focusing on its functionality of enforcing security (Authentication and Authorization) in dynamically deployed Virtual Organizations. The paper presents the overall architecture of the framework along with different security settings which FiVO can support within one Virtual Organization.
\end{abstract}

\section{Introduction}

Modern applications of Service Oriented Architectures or Grid computing are oriented on allowing distinct heterogenous organizations to share their resources in order to pursue some goal through advanced collaboration schemes supported by their IT infrastructures. The Grid idea introduced the concept of Virtual Organization, which abstracts the notion of organization into a virtual environment based on distributed computing infrastructures of organizations that want to collaborate. The idea of Virtual Organization allows these partners to define rules of cooperation in terms of authorization policies or SLA parameters which specify how their resources can and should be shared. The major problem that this idea is facing currently is the problem of very high administrative burden which is required to create and maintain a VOusing available tools. Additionaly no standard currently exists which would allow to actually specify the rules of cooperation by these organizations in a unified manner which then could be used to create the VO by means of configuring proper middleware as well as monitoring the VO operation in order to ensure that the rules which were agreed upon are properly respected. These issues are especially important in case of security focused dynamic environments where creation of $\mathrm{VO}$ cannot be delegated to regular system administrators. In order to support creation and management of such dynamic Virtual Organizations, the middleware must provide support 
for several issues, including resource sharing policy definition and enforcement, resource discovery and usage limited according to the VO policy and others.

In this paper we present our framework, called FiVO (Framework for intelligent Virtual Organization) that supports creation and management of dynamic Virtual Organizations with special focus on dynamic VO creation through contract negotiation and authorization of access to resources. The main feature of FiVO is the contract negotiation and management component, which enables coordinated establishment of agreement among partners who want to create a new Virtual Organization. The contract provides the information necessary for configuration of the VO in the system and allows for specification of both functional and non-functional parameters of the envisioned VO collaborations. Many problems related to ad-hoc creation of a VO are mostly related to heterogeneity of resources shared by VO members. Not only computer equipment is different, but also data formats, service descriptions, knowledge repositories. These issues require a method of mediation between $\mathrm{VO}$ members which is necessary to provide connectivity and make collaboration possible and efficient. With respect to VO deployment and contract enforcement we currently focus on security, i.e. authentication and authorization of requests to $\mathrm{VO}$ resources based on the contract statements. Other aspects will be developed as the framework evolves further.

\section{FiVO Architecture}

The architecture of FiVO is based on several goals. The first aim is to provide a unified semantic interface at the service level for discovery and management of all aspects relating to a Virtual Organization, including its members, agreements, goals, resources, data and services. This functionality is achieved through unification of metadata used to annotate these resources with the use of ontologies. FiVO is oriented strictly towards Service Oriented Architectures and Grid computing, thus assuming certain requirements on the infrastructures of organizations willing to participate in Virtual Organizations. These requirements however comply with the current trends in existing and emerging standards for information systems integration. FiVO supports both static and dynamic creation and deployment of Virtual Organizations aiming to pursue some goal, e.g. an emerging market opportunity. In order to enable using this solution in legacy environments the framework supports legacy information systems by providing adapters for existing middleware components. For instance MyProxy [1] and VOMS (Virtual Organization Membership Service) [2] adapters allow to configure these components after successful VO negotiation in order to support GSI based authentication according to the rules defined in the negotiated contract. Such features are of major importance, since legacy information systems and middleware components provide functionality crucial to VO management, especially at the low level and should be reused it in order to make integration between partners of a $\mathrm{VO}$ more feasible.

An important aspect of Virtual Organization lifecycle management supported by our framework is contract negotiation, which is a process involving all 
partners who want to participate in the emerging Virtual Organization. The contract itself is a set of statements which define terms (statements) related to the goal of the Virtual Organization on which all partners agree. Current FiVO provides framework for manual contract negotiation through a collaborative environment (either Protege plug-in or web-based). Negotiated contract, which is stored in the form of an ontology, is subsequently used to automatically configure the underlying security layer and QoS monitoring components in order to ensure contract enforcement during Virtual Organization operation. This enables organizations to state their commitments and requirements in an abstract way, while minimizing the burden on administrators who otherwise would have to configure all these parameters manually.

The essential contribution of FiVO to existing Virtual Organization management systems is in provision of a unified semantic interface for discovery and management of all aspects of a Virtual Organization (including its members, agreements, resources, goals, SLA's), allowing for dynamic inception through contract negotiation and automatic configuration of underlying middleware layer for contract enforcement during Virtual Organization operation. Figure 1 presents a sample deployment of the FiVO framework in some distributed environment. Four organizations can be seen, sharing their resources within the VO-1. FiVO component is deployed within each organization and is responsible

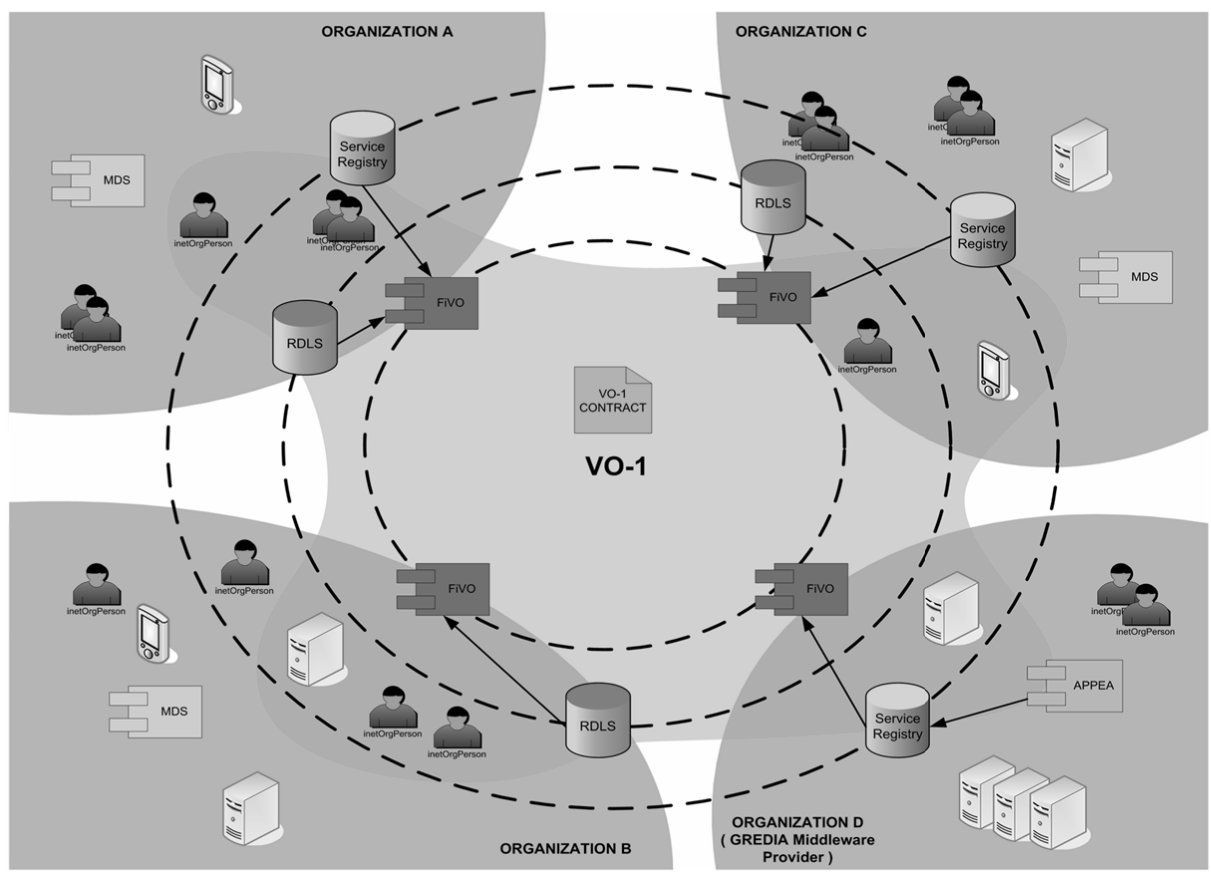

Fig. 1. Vision of the FiVO framework in a distributed environment 
for managing this organizations assets, assuming they are described semantically. During the contract negotiation phase, the FiVO components provide for the negotiators view on the resources of the participating organizations which can be used to create proper statements in the contract. After the contract is successfuly negotiated the semantic description of these resources along with the statements from the contract are used to configure proper middleware components. These descriptions can include such aspects of organization as its structure and business logic described in proper ontology as well as hardware, data and service resources available and provided for sharing with other organizations.

\section{Contract Negotiation}

In order to support the contract negotiation functionality we have defined a formal contract negotiation model and implemented it using Web Ontology Language (OWL) [3]. The vocabulary for the process of contract negotiation is defined by a set of ontologies providing common high-level terminology which can be further instantiated by proper domain level ontologies describing the domain and resources of organization participating within the negotiations. The negotiation process is controlled by a special Graphical User Interface developed for this purpose as a Protege [7] plug-in, see Figures 2] and 3. The formal model gives means to implement a negotiation framework which allows parties to define the rules of cooperation within a given VO. The formal model, as defined

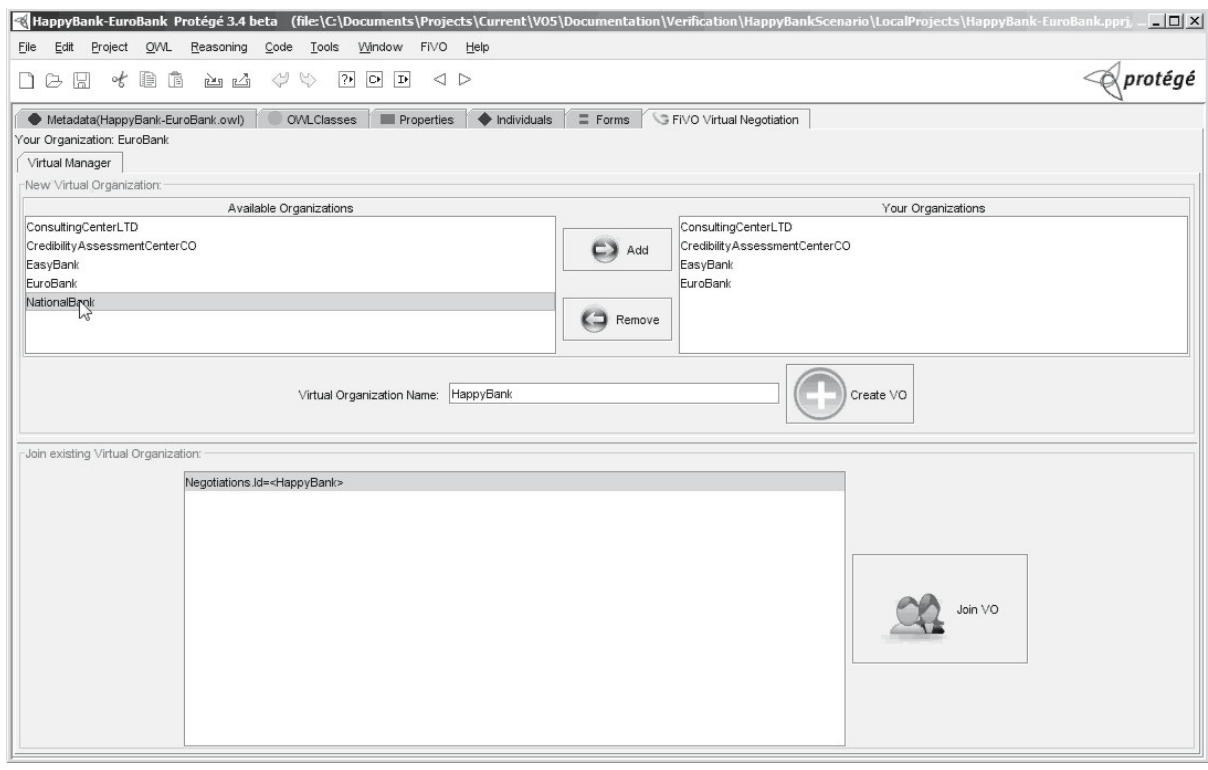

Fig. 2. Joining VO contract negotiations 


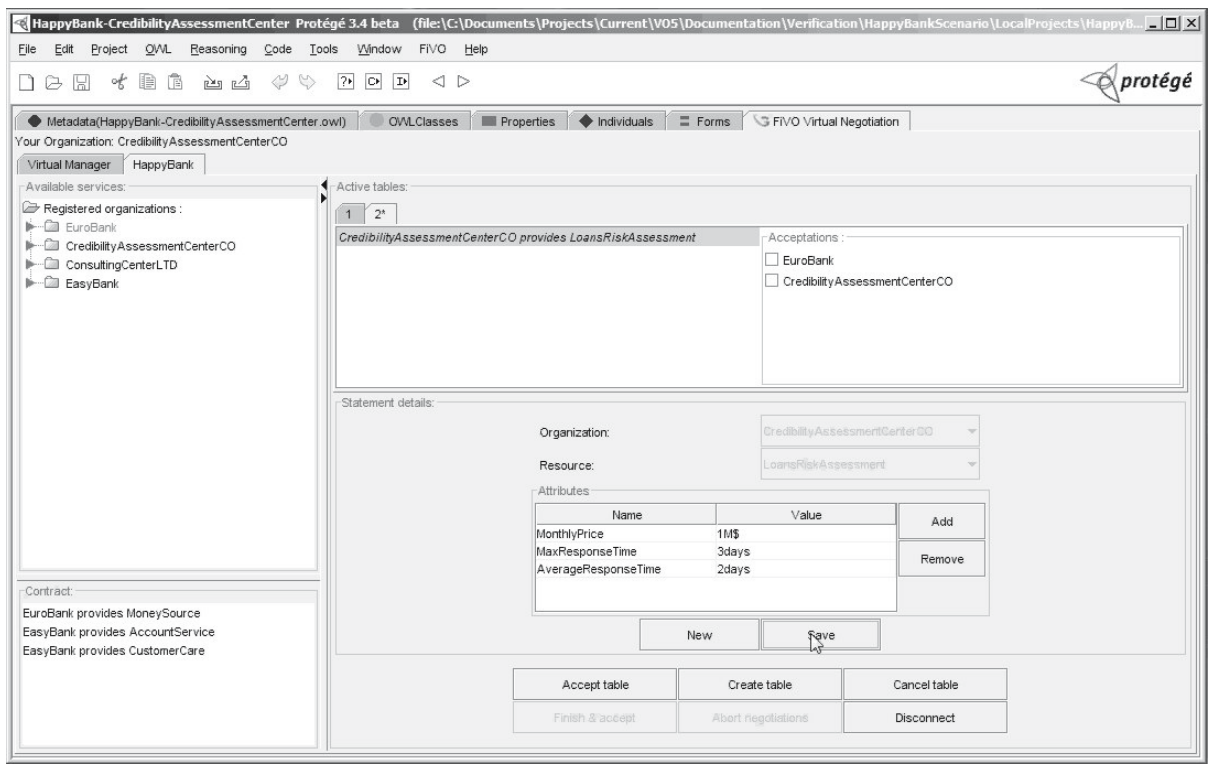

Fig. 3. Negotiating a statement within a negotiation table

in our previous work [6], defines the contract negotiation process in terms of messages exchanged between agents who control resources representing assets of their respective organizations. The negotiation process allows the negotiation of parts of the contract within negotiation tables, thus dividing the complete negotiation process into subnegotiations where only the parties which are directly concerned with the resources that particular statements address need to discuss. In the case of dynamic VO change, for instance when new resources should be added to the VO, contract amendment can be achieved by performing a new contract negotiation phase with the current contract as a starting point. In case the change in resources provided by the partners to the VO is not planned but is caused for instance by a service failure, the contract allows to detect that one of the partners does not fulfill his obligation to the VO and proper steps can be taken to deal with the problem.

\section{Semantically Supported VO Security}

The main motivation of supporting various security deployment configurations is to enable integration of heterogeneous IT infrastructures within a single VO. After the contract between the parties is negotiated, its rules are used to generate proper entries in MyProxy and VOMS components (i.e. set of roles and their mappings), and from then on FiVO login method uses these components in order to generate a X.509 proxy certificate for a given user. This credential can be then used anywhere within the Virtual Organization, e.g. presented to a Globus 


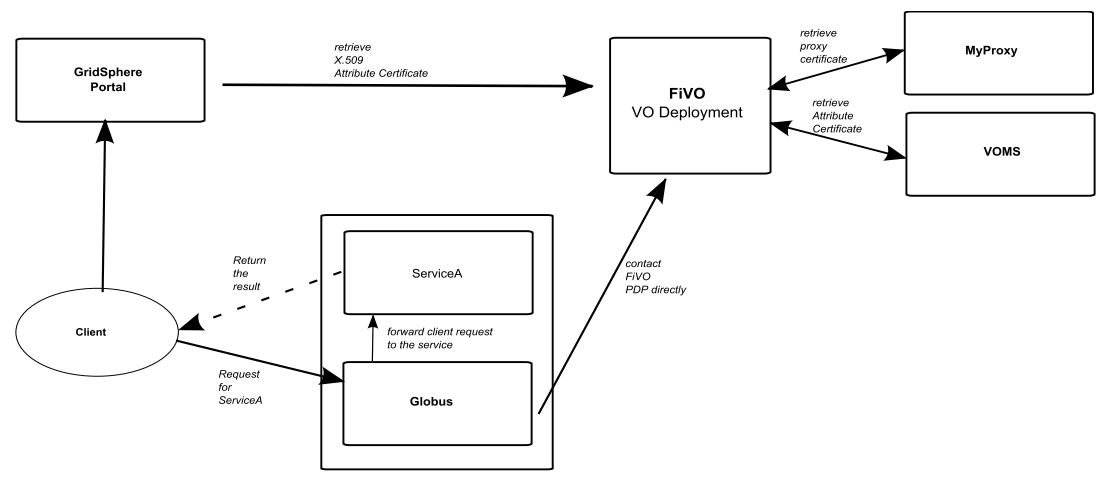

Fig. 4. Globus container and FiVO authentication and authorization

container or Apache web server. These containers will then contact FiVO PDP (Policy Decision Point) providing the role extracted from the proxy certificate presented by the user and receive an access decision.

Currently, FiVO supports 3 basic security deployments with Grid or SOA based environments, as depicted in Figures 4 5 and 6. In the first mode (Figure 44), when a Globus based WSRF service running in a Globus container is called by a remote client, proper Globus interceptors retrieve the Attribute Certificate from the users credential and send them to FiVO Authorization Service in order to get a AccessGranted/AccessDenied decision. The next mode (Figure 5) allows an Apache based web service (or any resource served by Apache server for that matter), a special Apache module - mod_authz_fivo - retrieves the credentials

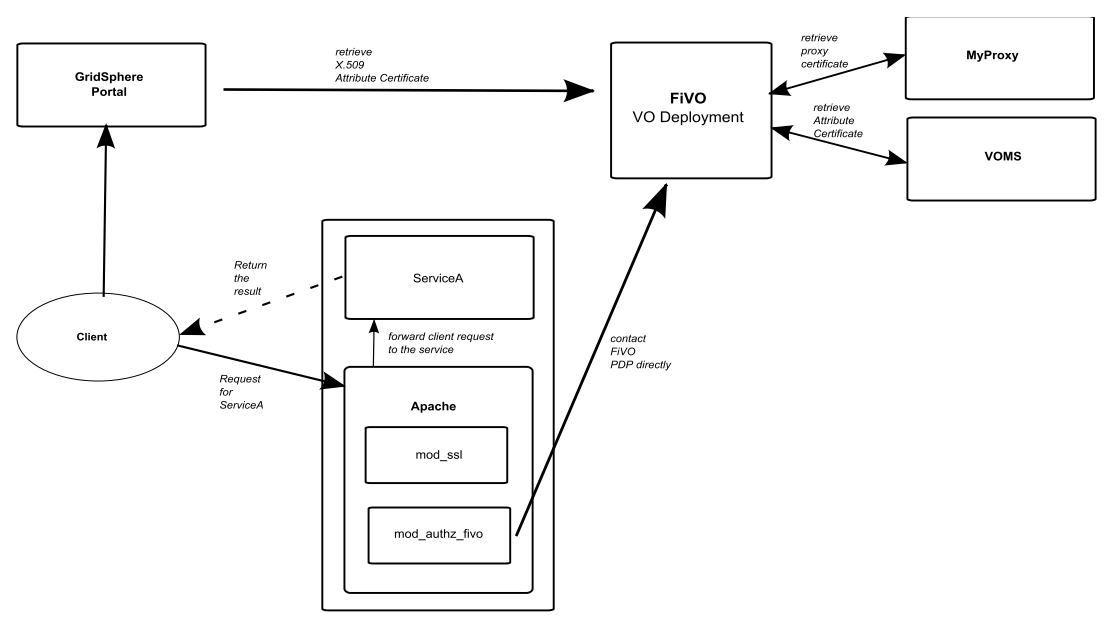

Fig. 5. Apache with FiVO authentication and authorization 


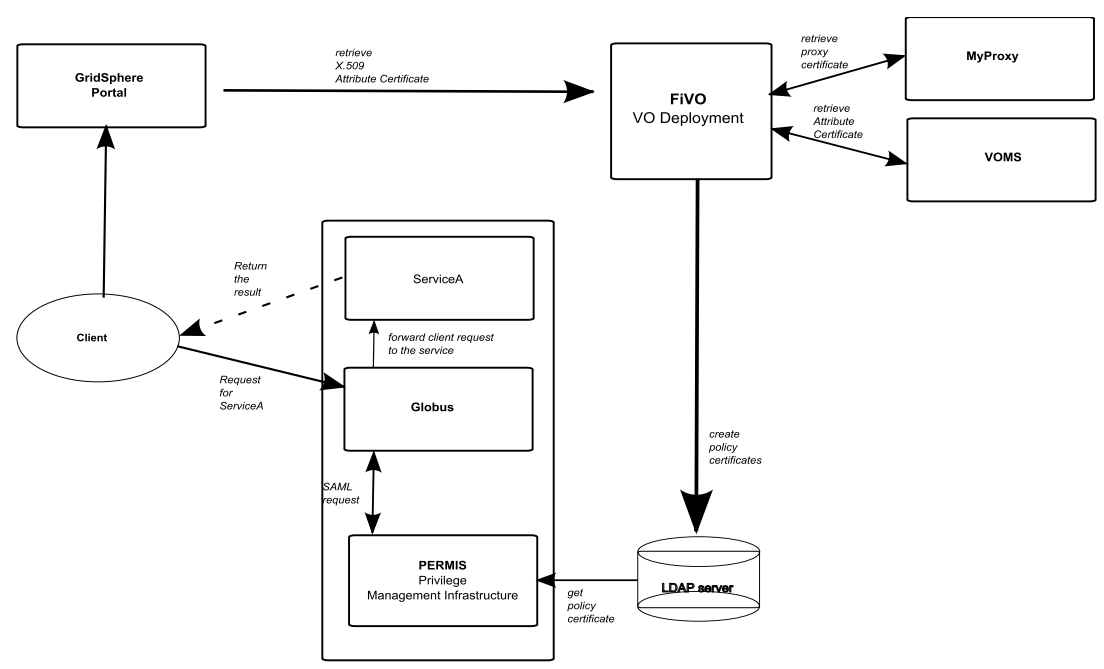

Fig. 6. Globus with FiVO authentication and PERMIS authorization

from the users certificate and contacts FiVO to get the authorization decision. Then it either continues the request processing or returns to the user HTTP Access Forbidden error. In the case of PERMIS (Figure 6), the situation is more complex. PERMIS 8] provides a standalone authorization service (PERMIS Policy Decision Point), however it depends on the LDAP service which contains proper information in terms of Attribute Certificates and Policies. FiVO works with PERMIS by feeding an LDAP service with proper policies, generated automatically from policies which are part of the VO contract. Currently there is an ongoing effort to integrate VOMS Attribute Certificates with PERMIS PDP in the framework of EU-IST VPMan project, so full integration of this approach will be possible as this project published their results. The authorization policies are defined as part of the ontology defining the contract between the partners of a VO, some sample policies are explained below. The first example presents a authorization rule allowing users with role BankManager to access a banking service. The rule assumes that the user has a role stored in a X509 credential, the action as reported by the Policy Enforcement Point (e.g. mod_authz_fivo Apache module) is Execute from a proper FiVO security ontology and the object is identified by the PEP as individual individual from the ontology describing the VO resources.

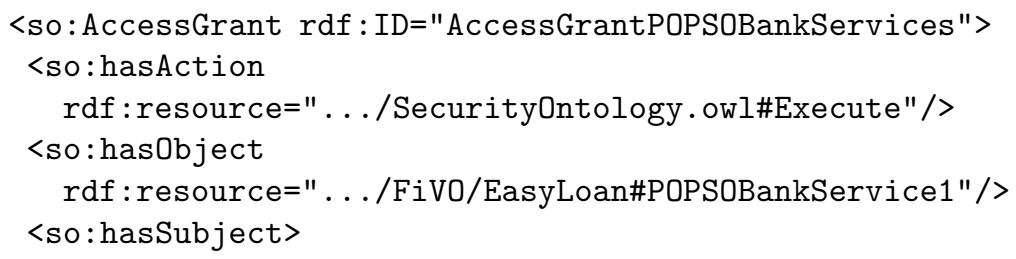




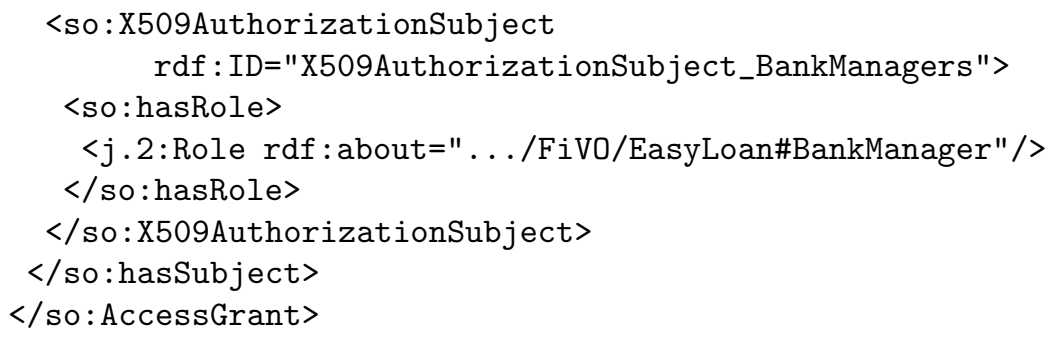

The second example presents an authorization rule allowing users with the role Editor to remove files from the system which have certain attributes, in this case location is equal to Iraq. The PEP is the data location service which checks with FiVO whether the user with role Editor can remove the files which have certain metadata attributes.

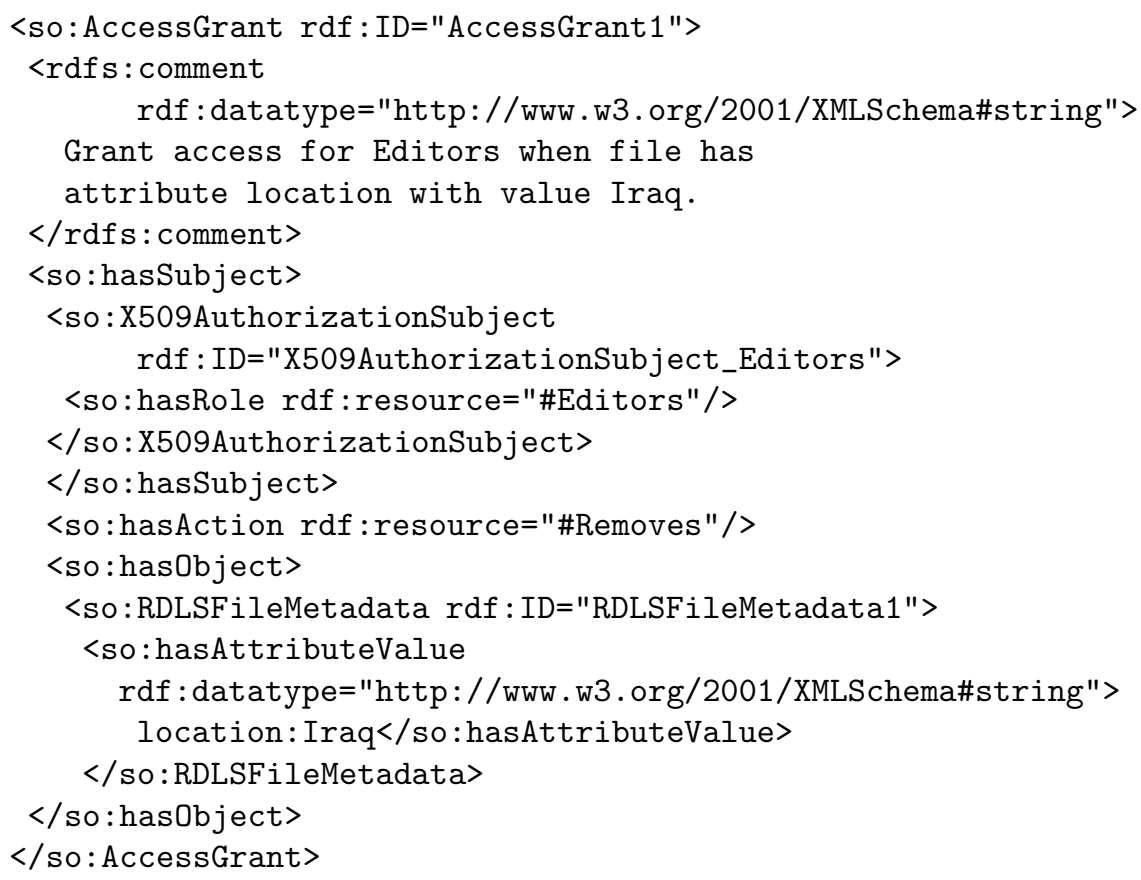

As can be seen, the use of ontological definition of policies allows for very flexible integration of various types of subjects.

\section{Related Work}

The enforcement of the contractual agreement is assumed to be handled by the underlying middleware, i.e. including both the security and SLA layers. Security aspects, especially with relation to dynamic Virtual Organization mainly 
should revolve around handling heterogeneity of middleware available in such distributed environments. Currently several solutions exist for both authentication and authorization issues which are essential to allow disparate organizations to cooperate together by their resources within interconnected IT infrastructures. The most common authentication systems include X.509 Public Key Infrastructure [9], Kerberos [10] and Shibboleth [1]. Additionally to authentication, the most popular authorization infrastructures for the SOA and Grid based systems include Virtual Organization Membership Service [2], Community Authorization Service [12, Akenti [13] and PERMIS 14. In [15] the authors describe integration of GSI and Shibboleth based security infrastructures within a VO based on an abstraction approach. In [16] authors present web-Pilarcos J2EE based agent framework for managing contract based Virtual Organizations. The contract itself is an object (J2EE EntityBean) and can be in several states, such as In-negotiation, Terminated etc. The proposed solution is not based on ontologies, and the metadata reasoning is mentioned only briefly. Unfortunately, none of these tools allows suffciently rich resource description framework that would allow to embrace the heterogeneity of infrastructures and environments found in Virtual Organizations, and more importantly their interoperability is very limited.

\section{Conclusions and Future Work}

In this paper we presented the motivation and architecture of the FiVO (Framework for Intelligent Virtual Organizations) which enables contract negotiation and management for Virtual Organizations which can be applied in heterogenous IT infrastructures such as demanding business settings. We believe that such functionality will foster the adoption of Virtual Organizations in commercial applications by simplifying the process of Virtual Organization inception and management of agreements specifying how the resources of each participating organizations should be shared among partners of a VO. The future work will be focused on provision of additional adapters allowing integration with other popular existing middleware solutions, such as configuring of monitoring components or setting up legacy software for use in a VO, and implementation of automatic contract negotiation agents based on the FiVO framework allowing fully autonomic VO deployment in case of emerging need for such coolaboration.

\section{Acknowledgments}

This research has performed done within the framework of EU IST FP6-34363 Gredia project. The authors would like to thank the whole project consortium for remarks and feedback. AGH University of Science and Technology grant nr 11.11.120.777 is also acknowledged. 


\section{References}

1. Basney, J., Humphrey, M., Welch, V.: The MyProxy online credential repository. Softw., Pract. Exper. 35(9), 801-816 (2005)

2. Alfieri, R., Cecchini, R., Ciaschini, V., dell'Agnello, L., Frohner, A., Lrentey, K., Spataro, F.: From gridmap-file to VOMS: managing authorization in a Grid environment. Future Generation Comp. Syst. 21(4), 549-558 (2005)

3. Antoniou, G., Van Harmelen, F.: Ontology Language: OWL. In: Handbook on Ontologies: International Handbook on Information Systems, pp. 67-92 (2004)

4. Kryza, B., Majewska, M., Pieczykolan, J., Slota, R., Kitowski, J.: Grid organizational memory - provision of a high-level grid abstraction layer supported by ontology alignment. Future Generation Comp. Syst., Grid Computing: Theory, methods and Applications 23(3), 348-358 (2007)

5. Kryza, B., Pieczykolan, J., Kitowski, J.: Grid organizational memory: A versatile solution for ontology management in the grid. In: Proc. of 2nd Intl. Conf. on eScience and Grid Computing, Amsterdam, Netherlands, December 4-6, 2006. IEEE Computer Society Press, Los Alamitos (2006)

6. Zuzek, M., Talik, M., Swierczynski, T., Wisniewski, C., Kryza, B., Dutka, L., Kitowski, J.: Formal Model for Contract Negotiation in Knowledge-Based Virtual Organizations. In: Bubak, M., et al. (eds.) ICCS 2008, Part III. LNCS, vol. 5103, pp. 409-418. Springer, Heidelberg (2008)

7. Knublauch, H., Fergerson, R.W., Noy, N.F., Musen, M.A.: The Protégé OWL Plugin: An Open Development Environment for Semantic Web Applications. In: McIlraith, S.A., Plexousakis, D., van Harmelen, F. (eds.) ISWC 2004. LNCS, vol. 3298, pp. 229-243. Springer, Heidelberg (2004)

8. Chadwick, D.W., Zhao, G., Otenko, S., Laborde, R., Su, L., Nguyen, T.: PERMIS: a modular authorization infrastructure. Concurrency and Computation: Practice and Experience 20(11), 1341-1357 (2008)

9. Landrock, P.: Public Key Infrastructure. In: van Tilborg, H. (ed.) Encyclopedia of Cryptography and Security. Springer, Heidelberg (2005)

10. Steiner, J.G., Neuman, C.N., Schiller, J.I.: Kerberos: An Authentication Service for Open Network Systems. In: Proc. of the Winter 1988 Usenix Conf., pp. 191-202 (1988)

11. Sinnot, R.O., Jiang, J., Watt, J.P., Ajayi, O.: Shibboleth-based Access to and Usage of Grid Resources. In: GRID, pp. 136-143. IEEE, Los Alamitos (2006)

12. Pearlman, L., Welch, V., Foster, I., Kesselman, C., Tuecke, S.: A community authorization service for group collaboration. In: Proc. Of Third IEEE International Workshop on Policies for Distributed Systems and Networks (POLICY 2002), pp. 50-59 (2002)

13. Johnston, W., Mudumbai, S., Thompson, M.: Authorization and Attribute Certificates for Widely Distributed Access Control. In: Proc. of IEEE 7th Int Workshops on Enabling Technologies: Infrastructure for Collaborative Enterprises (WET ICE), pp. 340-345 (1998)

14. Chadwick, D., Otenko, A., Ball, E.: Role-based access control with x.509 attribute certificates. IEEE Internet Computing 7(2), 62-69 (2003)

15. Kirchler, W., Schiffers, M., Kranzlmuller, D.: Harmonizing the Management of Virtual Organizations Despite Heterogeneous Grid Middleware: Assessment of Two Different Approaches. In: Proc. Cracow Grid Workshop, Krakow, Poland, October 2008 (March 2009) (in print)

16. Metso, J., Kutvonen, L.: Managing virtual organizations with contracts. In: Workshop on Contract Architectures and Languages, CoALa 2005 (2005) 